bly meet, so that the sharp edge of the one pipe is indented into the flat surface of the adjoining. The joints thus produced have been found capable of sustaining every pressure to which they have been applied, and the pipes themselves endure, without bursting, an expansive force of 3000 lbs. on the square inch.

The same principle is applied to cast-iron pipes for water-mains, \&c., the right and left hand screws being cast on each pipe, and the sockets are also cast in iron. In these cases a mill-board annular washer, soaked in oil, is placed between the flat ends of the two pipes, which are screwed together by the double nut, as in the former case.

Ibid.

\title{
A New Gas.
}

The "Censeur," of Lyons, states, that at one of the late sittings of the municipal council, a trial was made of a new portable gas, to which its inventor has given the name of "hydroluminous." The apparatus, says this journal, is very simple, and applicable to the smallest candlesticks, as well as to the largest and most splendid candelabra. The light it gives is very fine, and it is so portable that it can be carried about with the common hand candlestick.

Lond. Mech. Mag.

\section{Silver Plating as practiced at Sheffield. By Mr. Роттев, Jr.}

Plating on copper was first introduced in the year 1742, by Mr. Thomas Bolsover, a member of the Corporation of Cutlers, at Sheffeld, who, when repairing a knife-handle, composed partly of silver, and partly of copper, suddenly thought that it might be possible so to unite the two metals as to form a cheap substance, which, presenting an exterior of silver, might be used for the manufacture of several articles hitherto made entirely of that metal. It was not till about forty years after the introduction of Mr. Bolsover's plan, that the ornamentêd parts of plated articles, called mountings, were constructed of silver. This great improvement caused the manufacture of plated wares to become one of the staple trades of Sheffield.

There are two important features in the process of silver plating, the one a perfect adhesion of the two metals, the other a protection from wear of the prominent edges by friction.

The process of manufacturing plated articles may be described as follows:-

An ingot of copper being cast, and the surfaces carefully prepared by filing, so as to remove all blemishes, and a piece of silver, also having one surface perfectly cleaned, are tied together by means of iron wire. A paste of borax and water is then passed round the edges with a quill, and the mass being placed in a common air furnace, is heated to a proper temperature, which is ascertained by means of a small aperture in the door. As soon as the union of the two bodies is effected, which is known by the oozing of the metal when the fusion of the two metals has taken place, the bar is removed from 
the furnace. The quality of the silver used in this process is what is termed standard, containing about $18 \mathrm{dwts}$. of copper, to the pound troy. The effect of this alloy is to render the articles harder, and, consequently, more durable.

The ingot being thus prepared, the next operation is to form it into sheets, which is effected by passing the bar several times through large cylindrical rollers, generally moved by steam power; the lamination which the silver undergoes, during the operation of rolling, shows the perfect union of the two metals.

The dies for forming the ornamental parts of plated articles, consist of blocks of steel, on the face of which the pattern of the ornament is accurately drawn, after which the dies are moderately heated in an open fire, and then placed upon a leathern sand-bag; the die-sinker then proceeds to cut out the ornaments with hammer and chisel. When sunk to the proper depth, the surface of the sinking is dressed off, and prepared for the ornaments to be stamped in.

The stamping machine, of which a small working model was exhibited, consists of a vertical frame of iron, the uprights of which are formed with grooves in which the hammer, or drop, slides. The foundation of this machine consists of a square stone, on the upper surface of which is fixed an iron anvil, to which the uprights are firmly attached. The hammer is raised by a rope passing over a pulley fixed in the head-piece of the frame. The die is placed on the anvil immediately under the hammer, and kept in its proper position by screws. A luting of oil and clay is placed round the edge of the sink of the die, and melted lead is then poured into the cavity. When cool, the hammer is allowed to fall upon the lead, to which it firmly adheres by means of a plate roughed as a rasp, which is called the lick-up.

The silver used for the purpose of the mountings is also of standard quality, and is rolled to the required thickness; several pieces of the requisite size are then placed between pieces of copper of the same substance, and put upon the fuce of the die. The hammer is then raised, and allowed to fall gently upon them. This operation is continued for some time, gradually increasing the fall of the hammer, and diminishing the number of pieces struck, until they are forced to the bottom of the die; it is necessary occasionally to anneal the mountings.

The mounts being struck, as described, are now filled with solder, consisting of tin and lead, and afterwards secured by wires to the article to be ornamented, the body being covered with a mixture of glue and whiting, to prevent the solder from staining the surface; they are then soldered on by means of a gas blow-pipe.

The article is next boiled in a solution of pearl-ash, or soda, and scoured with fine Calais sand. The mounts are polished by a lathe, as in the case of silver articles, with rotten-stone and oil, then cleaned with whiting, and finished with rouge; a scratch-brush of brass wire is used for deadening the parts required, and the plain surfaces are burnished with tools of blood-stone, or steel, soap and water being used in this aperation, which is performed by women. 